\title{
Comparison of Efficacy and Side Effects of Azithromycin and Co-Amoxiclav in the Treatment of Acute Sinusitis in Adults: A Randomized Clinical Trial
}

\author{
Soroush Amani ${ }^{1}$, Ali Mohammadi-Najafabadi ${ }^{2} \&$ Ali Ahmadi ${ }^{3}$ \\ ${ }^{1}$ Kashani Hospital, department of otorangology, Shahrekord University of Medical Sciences, Shahrekord, Iran \\ ${ }^{2}$ Research Center for Modeling of Non Communicable Disease (NCD), Shahrekord University of Medical \\ Sciences, Iran \\ ${ }^{3}$ Health Faculty, Department of Epidemiology and Biostatisticics, Shahrekord University of Medical Sciences, \\ Shahrekord, Iran \\ Correspondence: Ali Ahmadi, Health Faculty, Department of Epidemiology and Biostatisticics, Shahrekord \\ University of Medical Sciences, Shahrekord, Iran. Tel: 98-913-383-4324. E-mail: aliahmadi2007@gmail.com
}

Received: January 13, 2016 Accepted: March 1, 2016 Online Published: March 23, 2016

doi:10.5539/gjhs.v8n11p178 URL: http://dx.doi.org/10.5539/gjhs.v8n11p178

\begin{abstract}
Background: Regarding high prevalence of common cold and sinusitis in Chaharmahal and Bakhtiari Province and the lack of studies on patients referring hospitals in this province, this study was conducted to determine and compare the efficacy and side effects of azithromycin and co-amoxiclav.

Methods: This study was a double-blinded randomized clinical trial. The study population of this clinical trial was consisted of 90 patients with acute sinusitis aged 12-65 years. At least two major criteria or one major criterion and two minor criteria with duration of at least 7 days and at most 28 days, were some of the sinusitis diagnostic criteria for inclusion of the patients into the study. The patients were examined for symptoms prior to and twice after the treatment. The treatment was administration with $\mathbf{5 0 0} \mathbf{~ m g}$ azithromycin tablet per day for three days in group 1 (G1) and $\mathbf{6 2 5} \mathbf{~ m g}$ co-amoxiclav tablet every $\mathbf{8} \mathbf{~ h}$ for 10 days in group 2 (G2). The data were analyzed by repeated measures analysis of variance, independent t-test, and chi-square in Stata software and $\mathrm{P}<$ 0.05 was considered significant.
\end{abstract}

Results: Mean \pm Standard deviation age of all patients was $32.14 \pm 9.91$ years. Out of 90 participants in the study, $50(55.56 \%)$ were male and the rest were female. The patients' symptoms were quantitatively similar in the two groups (22.3 \pm 2 and $22.2 \pm 1.9$ in G1 and G2, respectively) prior to the intervention. The score at second examination decreased to $3.7 \pm 2.1$ in $\mathrm{G} 1$ and to $9.4 \pm 2.7$ in $\mathrm{G} 2$, significantly different from that prior to intervention and between the two groups $(\mathrm{P}<0.001)$. Although the score decreased in the two groups at third examination, it was not significant in the two groups $(\mathrm{P}=0.652)$. The cure rate in azithromycin group was derived $87.5 \%$ and $88 \%$, and in co-amoxiclav group $32.4 \%$ and $83.3 \%$ at the days 5 and 10 of treatment, respectively. The most prevalent side effects in the two groups were diarrhea and nausea. The prevalence of diarrhea was obtained $21 \%$ and $33 \%$ in G1 and G2, respectively.

Conclusion: No significant difference in side effects was seen between the two groups. Although the cure was faster in the patients treated with azithromycin than those treated with co-amoxiclav, co-amoxiclav is still considered as adequately working against acute sinusitis bacterial pathogens. In view of this efficacy, co-amoxiclav seems to lead to no drug resistance and could be used to treat the patients.

Keywords: Azithromycin, co-amoxiclav, acute sinusitis

\section{Introduction}

There are more than a billion cases of viral and bacterial rhinosinusitis annually in the United States (Flint et al., 2014). It is estimated that it costs 5.8 billion $\$$ per year (Demury GP, Wald ER). Sinusitis has got many complications like mucous retention cyst, mucocele, inflammatory polyps and orbital complications. Orbital complications of paranasal sinus infection include: preseptal orbital edema, superior ophthalmic vein thrombosis, cavernous sinus thrombosis and blindness. 
Different treatments of sinusitis have been compared and reported since 1982, with little difference among them (von Sydow, Axelsson, \& Jensen, 1982). The clinical cure rate was reported $73.9 \%$ for azithromycin and $73.3 \%$ for co-amoxiclav in a study (Casiano, 1991). Cefpodoxime proxetil was demonstrated as clinically working as amoxicillin for treating acute bacterial maxillary sinusitis (von Sydow, Savolainen, \& Soderqvist, 1995). In view of the above studies and in a meta-analysis by Ioannidis et al., azithromycin was reported to lead to the clinical failure rates similar to the other antibiotics (Ioannidis, Contopoulos-Ioannidis, Chew, \& Lau, 2001). Clement reported that the total rate of clinical response was $87.5 \%$ for azithromycin and $83.7 \%$ for co-amoxiclav (Clement $\&$ de Gandt, 1998). The findings have indicated that antimicrobial therapy in short course ( $\leq 5$ days, $\leq 3$ days) for azithromycin could be as effective as and, occasionally, more effective than conventional therapies in long courses (10- to 14-day) (Guay, 2003).

Given cold climate, high altitude,high prevalence of sinusitis in Chaharmahal and Bakhtiari Province, lack of studies on patients referring hospitals in this topic in this province (Amani, Kheiri, \& Ahmadi, 2015), and the availability of azithromycin and co-amoxiclav as two drugs of choice, this study was conducted to determine and compare the efficacy and side effects of azithromycin and co-amoxiclav.

\section{Materials and Methods}

This study was a randomized clinical trial where both the allocator and examiner of the therapies were blinded. The study protocol was registered as IRCT201505106252N7 in Iranian Registry of Clinical Trials. The research adhered to Declaration of Helsinki, was approved by the Ethics Committee of the university (number : 92-6-24), and informed consent was obtained from all the participants.

Study population consisted of the patients with acute sinusitis aged 12-65 years. At least two major criteria or one major criterion and two minor criteria with duration of at least 7 days and at most 28 days have entered into the study. Pain or pressure in the face, facial fullness or congestion, nasal obstruction, nasal or postnasal purulent discharge, hyposmia or anosmia, and fever were considered as major criteria, and headache, halitosis, fatigue, toothache, ear pain or fullness, and cough as minor criteria. Chronic sinusitis, recurrent sinusitis, anatomic abnormalities, pregnancy or lactation, chronic diseases(renal failure,chronic liver and lung diseasis, diabetis mellitus and...) hypersensitivity to co-amoxiclav or azithromycin, congenital or acquired immunodeficiency, taking antibiotics one month before treatment, and the ages under 12 and over 65 years were considered as exclusion criteria.

90 patients referring the clinic were randomly entered the study. Patients were assigned to either of the groups of study by convenience randomization with equal probability. The patients were randomly divided into two groups that one group was treated with azithromycin and the other group was received co amoxiclav. The patients were examined for the symptoms and side effects such as diarrhea, nausea, and abdominal bloating at baseline and at the 5 th and $10^{\text {th }}$ day of treatment.

Pain or pressure in the face, facial fullness or congestion, nasal obstruction, nasal or postnasal purulent discharge, hyposmia or anosmia, fever, headache, halitosis, fatigue, toothache, ear pain or fullness, cough, and nasal hyperemia were assigned as the items of examination. All examinations were done by an otorhinolaryngologist. In addition to qualitative examination of the patients, the outcome of examinations was quantitatively recorded as $0-26$ for the above items in examination form. The patients who suffered more severe sinusitis and presented with more clinical symptoms were assigned higher scores. The patients who failed to adhere to therapeutic regimen or were not accessible throughout whole follow-up period were excluded from the study. The intervention consisted of $0.5 \%$ phenylephrine drop 3 drops 3 times a day for 2 days, nasal irrigation with normal saline 3 times a day, and one $500 \mathrm{mg}$ azithromycin tablet a day for 3 days in group 1, and $0.5 \%$ phenylephrine drop 3 drops 3 times a day for 2 days, nasal irrigation with normal saline 3 times a day, and one $625 \mathrm{mg}$ co-amoxiclav tablet every 8 hours for 10 days in group 2 . The data were analyzed by repeated measures analysis of variance (ANOVA), independent t-test, and chi square in STATA software. The level of significance was considered $<0.05$. The presuppositions of repeated measures ANOVA were investigated, and normal distribution and equality of variances (homoscedasticity) were established. Sphericity presupposition was not established and therefore Greenhouse-Geisser was used. 


\section{Results}

Of the 90 participants, $50(55.56 \%)$ were male and the rest were female. Of the 50 men, 25 underwent treatment with azithromycin and the rest with co-amoxiclav. Furthermore, of 40 women, 20 underwent treatment with azithromycin and the rest with co-amoxiclav. Mean \pm standard deviation age of all the studied patients was 32.14 \pm 9.91 years. Table 1 shows mean age of the patients for gender and treatment groups. The most prevalent complaint in the patients was nasal obstruction $(97.4 \%)$ followed by pain or pressure in face $(94.8 \%)$ and nasal or postnasal purulent discharge (94.8\%).

Table 1. Mean and standard deviation age of the patients

\begin{tabular}{llll}
\hline Treatment group & male & female & P value \\
\hline Azithromycin & $31.56 \pm 7.7$ & $31.55 \pm 7.73$ & 0.683 \\
Co-amoxiclav & $32.72 \pm 8.8$ & $29.2 \pm 7.23$ & 0.327 \\
Total & $32.14 \pm 9.91$ & $30.37 \pm 7.48$ & 0.353 \\
\hline
\end{tabular}

The frequency and percentage of the examined symptoms prior to and at two turns of examinations after treatment in all the patients and for either of treatment groups are shown in Table 2.

Table 2. Symptoms of disease prior to and after treatment for groups of study

\begin{tabular}{|c|c|c|c|c|c|c|c|c|c|c|c|c|}
\hline \multirow[t]{3}{*}{ Symptom } & \multicolumn{4}{|c|}{ Day 10 of intervention } & \multicolumn{4}{|c|}{ Day 5 of intervention } & \multicolumn{4}{|c|}{ Prior to intervention } \\
\hline & \multicolumn{2}{|c|}{ Group 2} & \multicolumn{2}{|c|}{ Group 1} & \multicolumn{2}{|c|}{ Group 2} & \multicolumn{2}{|c|}{ Group 1} & \multicolumn{2}{|c|}{ Group 2} & \multicolumn{2}{|c|}{ Group 1} \\
\hline & $\%$ & Frequency & $\%$ & Frequency & $\%$ & Frequency & $\%$ & Frequency & $\%$ & Frequency & $\%$ & Frequency \\
\hline $\begin{array}{l}\text { Pain or pressure in } \\
\text { face }\end{array}$ & 16.7 & 6 & 11.9 & 5 & 75 & 27 & 11.9 & 5 & 94.4 & 34 & 95.2 & 40 \\
\hline $\begin{array}{l}\text { Facial fullness or } \\
\text { congestion }\end{array}$ & 13.9 & 5 & 9.5 & 4 & 63.9 & 23 & 9.5 & 4 & 88.9 & 32 & 95.2 & 38 \\
\hline Nasal obstruction & $16 / 7$ & 6 & 11.9 & 5 & 66.7 & 24 & 11.9 & 5 & 97.2 & 35 & 97.6 & 41 \\
\hline $\begin{array}{l}\text { Nasal purulent } \\
\text { discharge }\end{array}$ & 16.7 & 6 & 11.9 & 5 & 63.9 & 23 & 11.9 & 5 & 94.4 & 34 & 95.2 & 40 \\
\hline Hyposmia & 11.1 & 4 & 9.5 & 4 & 41.7 & 15 & 9.5 & 4 & 72.2 & 26 & 69 & 29 \\
\hline Fever & 2.8 & 1 & 9.5 & 4 & 13.9 & 5 & 9.5 & 4 & 19.4 & 7 & 23.8 & 10 \\
\hline Headache & 11.1 & 4 & 11.9 & 5 & 55.6 & 20 & 14.3 & 6 & 88.9 & 32 & 85.7 & 36 \\
\hline Halsitosis & 8.3 & 3 & 9.5 & 4 & 47.2 & 17 & 9.5 & 4 & 58.3 & 21 & 52.4 & 22 \\
\hline Fatigue & 5.6 & 2 & 2.4 & 1 & 16.7 & 6 & 2.4 & 1 & 33.3 & 12 & 35.7 & 15 \\
\hline Toothache & $\mathbf{0}$ & $\mathbf{0}$ & 2.4 & 1 & 5.6 & 2 & 2.4 & 1 & 8.3 & 3 & 9.5 & 4 \\
\hline Cough & 5.6 & 2 & 0 & $\mathbf{0}$ & 19.4 & 7 & $\mathbf{0}$ & $\mathbf{0}$ & 25 & 9 & 26.2 & 11 \\
\hline $\begin{array}{l}\text { Ear pain or } \\
\text { fullness }\end{array}$ & 2.8 & 1 & 4.8 & 2 & 13.9 & 5 & 4.8 & 2 & 16.7 & 6 & 23.8 & 10 \\
\hline $\begin{array}{l}\text { Rhinoscopy } \\
\text { examination }\end{array}$ & 16.7 & 6 & 11.9 & 5 & 91.7 & 33 & 11.9 & 5 & 100 & 37 & 100 & 42 \\
\hline
\end{tabular}

The patients' symptoms were quantitatively similar in the two groups (22.3 \pm 2 and $22.2 \pm 1.9$ in group 1 and group 2 , respectively) prior to the intervention. The score at second examination decreased to $3.7 \pm 2.1$ in group 1 and to $9.4 \pm 2.7$ in group 2 , significantly different from that prior to intervention and between the two groups $(\mathrm{P}<0.001)$.

Although the score decreased in the two groups at third examination, it was not significant in the two groups $(\mathrm{P}=0.652)$. The cure rate in azithromycin group was derived $87.5 \%$ and $88 \%$, and in co-amoxiclav group $32.4 \%$ and $83.3 \%$ at the days 5 and 10 of treatment, respectively. Cure was defined the patients without any signs and 
symptoms of sinusitis. The most prevalent side effects in the two groups were diarrhea and nausea. The prevalence of diarrhea was obtained $21 \%$ and $33 \%$ in group 1 and group 2 , respectively.

\section{Discussion}

In this study, the efficacy of two pharmacological regimens on treatment of acute sinusitis was assessed .Because culture of the sinus secretion is aggressive and has risk of iatrogenic infection and also being expensive and requires a minimum 72 hours for implementation,thus diagnosis and treatment of bacterial sinusitis are often fulfilled clinically and empirical. In different studies, the patients' purulent discharge, nasal obstruction and fullness, and pressure and pain in face have been considered as main criteria for diagnosing acute sinusitis (Lemiengre, van Driel, Merenstein, Young, \& De Sutter, 2012; Riechelmann, Giotakis, \& Kral, 2013; Nematian-Samani, \& Mosges, 2014; Smith, Ference, Evans, Tan, Kern, \& Chandra, 2015). In a study the efficacy and side effects of $500 \mathrm{mg}$ azithromycin taken daily for 3 and 6 days were compared with those of co-amoxiclav 500/125 mg taken 3 times a day for 10 days with regard to treatment of acute sinusitis in adults . The efficacy of azithromycin was obtained $88.8 \%$ and $89.3 \%$ for 3 and 6 days, respectively, and that of co-amoxiclav was reported $84.9 \%$ (Henry, Riffer, Sokol, Chaudry, \& Swanson, 2003). In our study, the cure rate in azithromycin group was $88 \%$ and in co amoxiclav group was $83 / 3 \%$ at tenth day, this shows our findings are in line with this study.

In another study $4.9 \%$ of patients in the azithromycin group and $8.1 \%$ in the amoxicillin group reported side effects. Most side effects were gastrointestinal and were reported by 4 of 5 ( 3 amoxicillin, 1 azithromycin) patients. All side effects were mild, and only minor abnormalities in laboratory findings were reported in both groups. No patient declined to participate in the study because of side effects. In this study, a 5-day course (one dose a day) of azithromycin was demonstrated to have efficacy, safety, and tolerability, similar to those of a 10-day course (3 doses a day) of amoxicillin for the treatment of acute bacterial sinusitis (Casiano, 1991), similarly in our study the side effects were gastrointestinal but the prevalence of side effects were more than Casiano's study.

In a per protocol analysis, 117 patients in the cefpodoxime group and 113 in the amoxicillin group were compared for clinical efficacy. The clinical response rates were derived $96 \%$ and $91 \%$, respectively, and the corresponding figures in the intent-to-treat analysis were 130 and 128 patients, with clinical response rates of $93 \%$ and $88 \%$, respectively. Cefpodoxime proxetil was clinically found as effective as amoxicillin for the treatment of acute bacterial maxillary sinusitis. It was more effective on resolving Haemophilus influenzae and more efficient in enhancing the radiological score. Side effects were reported in $20 \%$ of cefpodoxime-treated patients and $16 \%$ of amoxicillin-treated patients with no statistically significant difference between the groups (von Sydow, Savolainen, \& Soderqvist, 1995).

Microbiological responses to both drugs were acceptable, and only 5 patients in each group had a persistent infection after treatment. Both drugs were well tolerated and caused similar adverse events, which were mostly gastrointestinal. Azithromycin and co-amoxiclav were similar for efficacy and tolerability, while azithromycin's shorter simpler and dosing regimen may be preferable with regard to compliance and cost (Clement \& de Gandt, 1998).

A meta-analysis of randomized controlled trials compared 3-5 days of azithromycin with other antibiotics conventionally taken in longer courses for the treating upper respiratory tract infections. The study consisted of 19 comparisons (3421 patients) for acute otitis media, 11 comparisons (including 1742 patients) for acute sinusitis, and 16 comparisons (including 2447 patients) for acute pharyngitis; azithromycin had comparable clinical failure rates to the other antibiotics. There were no significant differences in bacteriological outcomes, as well. Azithromycin was withdrawn because of adverse effects by only 37 of $4870(0.8 \%)$ patients. Short courses of azithromycin are as effective as longer courses of other antibiotics for upper respiratory tract infections. Despite easy dosing, this regimen for the treatment of these common infections has been increasing in cost, where often no other antibiotic may be prescribed (Ioannidis, Contopoulos-Ioannidis, Chew, \& Lau, 2001).

In a study, a significantly quicker healing of signs and symptoms of sinusitis has been noted in the azithromycin-treated patients. By the end of treatment (days 10-12), $95 \%$ and $74 \%$ of the patients in the azithromycin and amoxicillin/clavulanate groups were treated (Klapan, Culig, Oreskovic, \& Matrapazovski, Radosevic, 1999). There is a difference in result between our study and this study, it can be related to the number of patients, on the other hand we used different pharmacological products that it can be the another reason of this contrast.

The treatment results of 27 various treatment methods were compared in 1320 cases of acute maxillary sinusitis (2039 maxillary sinuses). The treatment regimens were not found to be much different in therapeutic effect, 
irrespective of treatment with antral drainage alone, antibiotics alone or their combination (von Sydow, Axelsson, \& Jensen, 1982).

\section{Conclusion}

The cure rate in azithromycin group was derived $87.5 \%$ and $88 \%$, and in co-amoxiclav group $32.4 \%$ and $83.3 \%$ at the day 5 and 10 of treatment, respectively. Diarrhea and nausea were the most prevalent side effects in the two groups. The prevalence of diarrhea was $21 \%$ in group 1 and $33 \%$ in group 2 respectively and no significant difference in side effects was seen between the two groups of study. Although the patients treated with azithromycin were cured more quickly than those treated with co-amoxiclav, co-amoxiclav is still known as being satisfactorily effective on bacterial pathogens of acute sinusitis. Because of this efficacy, co-amoxiclav seems to cause no drug resistance and could be used to cure the disease.

\section{Acknowledgements}

Hereby, we thank the university authorities, patients and all those who supported us in implementation and completion of this research.

\section{Conflict of Interest}

The authors declare that there is no conflict of interests regarding the publication of this paper.

\section{References}

Amani, S., Kheiri, S., \& Ahmadi, A. (2015). Honey versus diphenhydramine for post-tonsillectomy pain relief in pediatric cases: A randomized clinical trial. Journal of Clinical and Diagnostic Research, 9(3), SC01-SC4.

Clement, P. A., \& de Gandt, J. B. (1998). A comparison of the efficacy, tolerability and safety of azithromycin and co-amoxiclav in the treatment of sinusitis in adults. The Journal of international medical research, 26(2), 66-75. PubMed PMID: 9602984.

Demury, G. P., \& Wald, E. R. (2010). Sinusitis. In G. L. Mandel, J. E. Bennett, \& R. Dolin, Principles and practice of infectious disease (7th ed.). Philadelphia: cherchil Livingston.

Flint, Paul W., et al. (2014). Cummings otolaryngology-head and neck surgery. Elsevier Health Sciences.

Guay, D. (2003). Short-course antimicrobial therapy of respiratory tract infections. Drugs, 63(20), 2169-84. PubMed PMID: 14498754. http://dx.doi.org/10.2165/00003495-200363200-00002

Henry, D. C., Riffer, E., Sokol, W. N., Chaudry, N. I., \& Swanson, R. N. (2003). Randomized double-blind study comparing 3- and 6-day regimens of azithromycin with a 10-day amoxicillin-clavulanate regimen for treatment of acute bacterial sinusitis. Antimicrobial agents and chemotherapy, 47(9), 2770-4. PubMed PMID: 12936972. PMCID: PMC182642. http://dx.doi.org/10.1128/AAC.47.9.2770-2774.2003

Ioannidis, J. P., Contopoulos-Ioannidis, D. G., Chew, P., \& Lau, J. (2001). Meta-analysis of randomized controlled trials on the comparative efficacy and safety of azithromycin against other antibiotics for upper respiratory tract infections. The Journal of antimicrobial chemotherapy, 48(5), 677-89. PubMed PMID: 11679557. http://dx.doi.org/10.1093/jac/48.5.677

Klapan, I., Culig, J., Oreskovic, K., Matrapazovski, M., \& Radosevic, S. (1999). Azithromycin versus amoxicillin/clavulanate in the treatment of acute sinusitis. American journal of otolaryngology, 20(1), 7-11. PubMed PMID: 9950107.

Lemiengre, M. B., van Driel, M. L., Merenstein, D., Young, J., \& De Sutter, A. I. (2012). Antibiotics for clinically diagnosed acute rhinosinusitis in adults. The Cochrane database of systematic reviews, 10, CD006089. PubMed PMID: 23076918. http://dx.doi.org/10.1002/14651858.CD006089.pub4

Nematian-Samani M, Mosges R. [Rhinitis - causes, complications, therapy]. MMW Fortschritte der Medizin, 13, 156. PubMed PMID: 25552018. http://dx.doi.org/10.1007/s15006-014-3483-y

Riechelmann, H., Giotakis, A., \& Kral, F. (2013). [Acute rhinosinusitis in adults--EPOS 2012 Part II]. Laryngorhino- otology, 92(11), 763-76. PubMed PMID: 24174339. http://dx.doi.org/10.1055/s-0033-1355415

Smith, S. S., Ference, E. H., Evans, C. T., Tan, B. K., Kern, R. C., \& Chandra, R. K. (2015). The prevalence of bacterial infection in acute rhinosinusitis: A Systematic review and meta-analysis. The Laryngoscope, 125(1), 57-69. PubMed PMID: 24723427. Pubmed Central PMCID: PMC4192102. http://dx.doi.org/10.1002/lary.24709

von Sydow, C., Axelsson, A., \& Jensen, C. (1982). Acute maxillary sinusitis--a comparison between 27 different treatment modes. Rhinology, 20(4), 223-9. PubMed PMID: 6187058. Epub 1982/12/01. eng. 
von Sydow, C., Savolainen, S., \& Soderqvist, A. (1995). Treatment of acute maxillary sinusitis--comparing cefpodoxime proxetil with amoxicillin. Scandinavian journal of infectious diseases, 27(3), 229-34. PubMed PMID: 8539546. http://dx.doi.org/10.3109/00365549509019014

\section{Copyrights}

Copyright for this article is retained by the author(s), with first publication rights granted to the journal.

This is an open-access article distributed under the terms and conditions of the Creative Commons Attribution license (http://creativecommons.org/licenses/by/3.0/). 